\title{
Fatores intervenientes na integridade cutânea de recém-nascidos em Unidade de Cuidado Intermediário Neonatal Convencional
}

\author{
Intervening factors in the cutaneous integrity of the newborn in a Conventional Neonatal Intermediate Care Unit
}

Factores que intervienen en la integridad cutánea de recién nacidos en Unidad de Cuidados Intermedios

Neonatales Convencionales

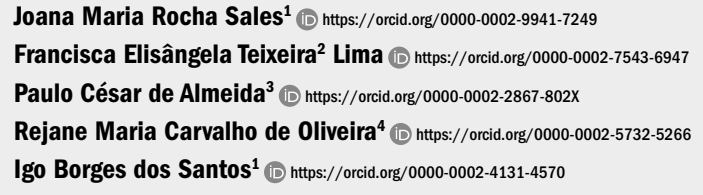

\section{Resumo}

Objetivo: Avaliar os fatores que interferem na integridade da pele de recém-nascidos hospitalizados.

Métodos: Pesquisa descritiva, transversal, de abordagem quantitativa, realizada com 46 recém-nascidos, em uma unidade de cuidado intermediário neonatal convencional de hospital público terciário, em Fortaleza-Ceará, Brasil, de agosto a outubro de 2020. Dados coletados com a Escala de Condição da Pele do Recém-Nascido e 0 formulário de dados sociodemográficos e clínicos do recém-nascido, os quais foram analisados pela estatística analítica, utilizando-se dos testes binomial, Qui-quadrado, razão de verossimilhança e de Fisher-Freeman-Halton. Resultados: Verificou-se que $27(58,7 \%)$ recém-nascidos apresentaram estado da pele alterado, cujos fatores intervenientes, com diferença significativa $(p<0,05)$, foram: recém-nascido prematuro, baixo peso ao nascer, pequeno para idade gestacional, distúrbio geniturinário e filho de mãe com idade $<30$ anos.

Conclusão: Constatou-se elevada alteração na condição cutânea de recém-nascidos com fatores maternos e neonatais associados a esse desfecho.

\section{Abstract}

Objective: Evaluate the factors that interfere with the skin integrity of the hospitalized newborn.

Methods: Descriptive, cross-sectional research, quantitative approach carried out with 46 newborns in a conventional neonatal intermediate care unit of a public tertiary hospital, in Fortaleza-Ceará, from August to October 2020. The data was collected using the Scale of Skin Condition of the Newborn and sociodemographic and clinical forms. It was then statistically analyzed using the binomial, Chi-square, likelihood ratio and Fisher-Freeman- Halton tests. Results: It was found that $27(58.7 \%)$ newborns had altered skin, whose intervening factors with significant difference $(p<0.05)$ were: premature newborn, low birth weight born, small for gestational age, genitourinary disorder and child of a mother aged $<30$ years.

Conclusion: Maternal and neonatal factors are associated with high changes in the cutaneous condition of the newborn.

\section{Resumen}

Objetivo: Evaluar los factores que interfieren con la integridad de la piel del recién nacido hospitalizado. Métodos: Estudio descriptivo, transversal con abordaje cuantitativo realizado con 46 recién nacidos en una unidad de cuidados intermedios neonatales convencional de un hospital terciario público, en Fortaleza-Ceará, de agosto a octubre de 2020. Los datos fueron recolectados con la Escala de Condición de la Piel del Recién Nacido. y el formulario de datos sociodemográficos y clínicos del recién nacido y analizados mediante estadísticas analíticas utilizando pruebas binomiales, chi-cuadrado, razón de verosimilitud y de Fisher-Freeman- Halton.

Resultados: Se encontró que $27(58,7 \%)$ recién nacidos presentaron alteración del estado de la piel, cuyos factores intervinientes, con diferencia significativa $(p<0,05)$, fueron: recién nacido prematuro, bajo peso al nacer, pequeño para la edad gestacional, trastorno genitourinario y hijo de una madre $<30$ años.

Conclusión: Hubo un gran cambio en la condición de la piel del recién nacido con factores maternos y neonatales asociados con este resultado.

\section{Descritores}

Enfermagem pediátrica; Pele; Recémnascido; Fatores de risco

Keywords

Pediatric nursing; Skin; Infant, Newborn; Risk factors

\section{Descriptores}

Enfermería pediátrica; Piel; Recién Nacido; Factores de riesgo

\section{Como citar:}

Sales JM, Lima FE, Almeida PC, Oliveira RM, Santos IB. Fatores intervenientes na integridade cutânea de recém-nascidos em Unidade de Cuidado Intermediário Neonatal Convencional. Rev Soc Bras Enferm Ped. 2021;21(1):44-50.

\footnotetext{
${ }^{1}$ Escola de Saúde Pública do Ceará, Fortaleza, CE,Brasil.

${ }^{2}$ Departamento de Enfermagem, Universidade Federal do Ceará, Fortaleza, CE, Brasil.

${ }^{3}$ Universidade Estadual do Ceará, Fortaleza, CE, Brasil.

${ }^{4}$ Universidade de Fortaleza, Fortaleza, CE, Brasil.

Conflitos de interesse: nada a declarar.

Submetido: 31 de Março de 2020 | Aceito: 30 de Julho de 2021

Autor correspondente: Joana Maria Rocha Sales | E-mail: joanamr@@yahoo.com.br

DOI: http://dx.doi.org/10.31508/1676-3793202100006
} 


\section{Introdução}

O período neonatal é considerado a faixa etária pediátrica com maior risco de mortalidade, por apresentar vulnerabilidades oriundas dos riscos ambientais, socioeconômicos e biológicos inerentes ao primeiro mês de vida. ${ }^{(1)}$

No panorama internacional, diversas causas estão associadas à mortalidade neonatal, dentre as quais, destacam-se as complicações associadas à prematuridade e ao parto, além daquelas oriundas de sepse e anormalidades congênitas. ${ }^{(1)}$ Ao considerar o cenário brasileiro, enumerar os fatores determinantes para óbitos neonatais, constata-se que a maioria também esteve relacionada com a prematuridade, característica presente em $68 \%$ das mortes, além do baixo peso ao nascer presente em $70 \%$ desses desfechos. ${ }^{(2)}$

Desse modo, observa-se que a transição fetal-neonatal determina críticas mudanças funcionais no organismo de recém-nascidos ( $\mathrm{RN}$ ) que o torna vulnerável e que afetam, dentre outros órgãos e sistemas, $\mathrm{o}$ revestimento cutâneo submetido a um contínuo processo de maturação. Dentre as funções, a pele exerce importante função de barreira contra infecções sistêmicas, desidratação e intoxicação, além de possibilitar a termorregulação e proteção física e imunológica ao organismo do RN. . $^{(3,4)}$

À vista disso, no que se refere à população neonatal hospitalizada, somam-se às fragilidades cutâneas inerentes ao período neonatal os diversos procedimentos compreendidos no cuidado. A assistência ao $\mathrm{RN}$ no contexto hospitalar envolve o uso de adesivos, eletrodos, sensores, cateteres de terapia endovenosa, dentre outros dispositivos. Tal fato aumenta substancialmente o risco de lesão de pele nessa população. ${ }^{(5)}$

Esse evento adverso está presente em mais da metade dos recém-nascidos em cuidados intensivos. Contudo, apesar da taxa de incidência de lesões ser menor nas unidades de cuidados intermediários, esses ambientes também oferecem risco para integridade cutânea do RN. ${ }^{(6,7)}$ Ao considerar a imaturidade do sistema imunológico do RN, a pele como barreira de proteção contra patógenos e o risco de infecção inerente ao ambiente hospitalar, vê-se a relevância de avaliar sistematicamente as condições da pele do RN hospitalizado e os fatores que podem induzir o surgimento de disfunções cutâneas, de modo a identificar precocemente alterações na pele, com a consequente intervenção em tempo hábil, para evitar infecções e septicemia. ${ }^{(8)}$

$\mathrm{Na}$ assistência hospitalar, a atuação do enfermeiro, como membro da equipe multiprofissional, é de extrema importância para o monitoramento da pele e a preservação da integridade cutânea neonatal. Este profissional é responsável direto pela manutenção do equilíbrio térmico, promoção de higiene e conforto, realização de curativos e procedimentos invasivos, dentre outros cuidados, devendo constantemente avaliar a pele do RN, para prevenção de lesões e consequente excelência do cuidado prestado. ${ }^{(5,8)}$

Diante dessas considerações e da importância da manutenção da integridade cutânea para melhor prognóstico do RN e qualificação do cuidado de enfermagem, buscou-se responder aos questionamentos: quais fatores interferem na integridade da pele do RN em unidades de cuidados intermediários convencionais? Quais as condições da pele do RN hospitalizado?

Frente ao exposto, objetivou-se avaliar os fatores intervenientes na integridade da pele de $\mathrm{RN}$ internados em unidade de cuidados intermediários convencionais.

\section{Métodos}

Estudo descritivo, transversal e com abordagem quantitativa, desenvolvido em Unidade de Cuidado Intermediário Neonatal Convencional (UCINCo) de hospital público pediátrico de grande porte, da atenção terciária do governo do estado do Ceará, na cidade de Fortaleza.

A UCINCo avaliada dispõe de 23 leitos estruturados em quatro enfermarias, com perfis distintos de internação: neonatologia geral; prematuridade; cirurgia neonatal; e, por fim, uma enfermaria destinada aos recém-nascidos infectados. Do universo de recém-nascidos internados durante o período da coleta de dados, considerou-se como critério de inclusão as crianças até 28 dias de vida, abordando, desta forma, apenas o período neonatal; e, como critério de exclusão, os recém-nascidos cujos prontuários não apresentavam a totalidade dos dados a serem analisados. Logo, a amostra para o presente estudo foi composta por 46 recém-nascidos.

A coleta de dados, desenvolvida no período de agosto a outubro de 2020, ocorreu mediante o preen- 
chimento do formulário de dados sociodemográficos e clínicos coletados a partir das informações presentes no prontuário, que considerou as seguintes variáveis: iniciais do $\mathrm{RN}$, idade gestacional (IG), peso ao nascer, sexo, Apgar, data de nascimento, dias de vida, idade materna, procedência, diagnóstico clínico, via de administração e medicações em uso, suporte nutricional e de oxigenação, uso de acesso intravenoso e fototerapia. Posteriormente, aplicou-se a Escala de Condição da Pele do Recém-Nascido (ECPRN), mediante a inspeção visual da pele do $\mathrm{RN}$, para observar presença de drenos e ostomias e existência de lesões de pele, a fim de ponderar tipo, local e características morfológicas.

A ECPRN é um instrumento de avaliação da pele do recém-nascido validado e adaptado para o contexto brasileiro. Este instrumento se subdivide em três aspectos para avaliação: secura, eritema e ruptura/ lesão. Para cada item, atribui-se uma pontuação que varia de um a três. Desta forma, o escore final obtido pertence ao intervalo de três a nove pontos, sendo três o estado ideal da pele e nove a pior condição de pele. Assim, classificou-se como estado da pele ideal o RN com pontuação igual a três pontos; e estado da pele alterado, o RN com pontuação superior a três pontos. ${ }^{(9)}$

Analisaram-se as associações entre a condição de pele com as características maternas e do RN; com os aspectos clínicos e de dispositivos terapêuticos em uso durante a internação; e com outros fatores relacionados. Para tanto, empregaram-se os testes binomial, Qui-quadrado, razão de verossimilhança e de Fisher-Freeman-Halton. A força dessa associação foi calculada pela razão de chance e pelo intervalo de confiança de $95 \%$. Para as análises inferenciais, consideraram-se estatisticamente significantes se $p<0,05$. Os dados foram processados no SPSS 20.0, licença número 10101131007.

Em conformidade aos princípios éticos referentes às pesquisas científicas que envolvem seres humanos, o estudo foi aprovado pelo Comitê de Ética em Pesquisa, conforme parecer $n^{0}$ 4.202.031/2020.

\section{Resultados}

Do total de recém-nascidos participantes, 27(58,7\%) apresentaram estado da pele alterado, com esco- re superior a três pontos na ECPRN. Ao analisar as dimensões abordadas, verificou-se que 40(87,0\%) recém-nascidos apresentaram pele sem descamação visível, 31(67,4\%) sem eritema e 34(73,9\%) não tinham lesão ou ruptura na pele (Tabela 1). No quesito secura, não houve RN classificado com pele muito seca que evidenciasse rachaduras/fissuras. Na tabela 1, dispõem-se as variáveis maternas, gestacionais e neonatais, segundo o estado da pele apresentado pelo RN. Dentre os fatores neonatais e maternos que intervinham no estado da pele, a IG, o peso ao nascer, o crescimento intrauterino e a idade materna obtiveram associação significativa.

Tabela 1. Dados referentes à aplicação da Escala de Condição de Pele do Recém-Nascido (ECPRN) $(n=46)$

\begin{tabular}{lc}
\hline Variáveis & $\mathrm{n}(\%)$ \\
\hline Secura & $40(87,0)$ \\
$\quad$ Pele normal (1 ponto) & $6(13,0)$ \\
$\quad$ Descamação visível (2 pontos) & \\
\hline Eritema & $31(67,4)$ \\
$\quad$ Não há evidência de eritema (1 ponto) & $12(26,1)$ \\
$\quad$ Eritema visível (<50\% da superfície corporal) (2 pontos) & $3(6,5)$ \\
$\quad$ Eritema visível ( $\geq 50 \%$ da superfície corporal) (3 pontos) & $34(73,9)$ \\
\hline Ruptura/Lesão & $10(21,7)$ \\
$\quad$ Nenhuma visível (1 ponto) & $2(4,4)$ \\
$\quad$ Pequenas, em áreas localizadas (2 pontos) & $19(41,3)$ \\
$\quad$ Extensa (3 pontos) & $27(58,7)$ \\
\hline Pontuação total & \\
$\quad$ Estado da pele ideal (= 3 pontos) & \\
$\quad$ Estado da pele alterado (> 3 pontos) &
\end{tabular}

Recém-nascido com IG $<37$ semanas (IC 95\% 1,4 $-2,7 ; \mathrm{p}=0,004)$ e RN com peso ao nascer $<2.500$ gramas (IC 95\% 1,5 - 3,1; p = 0,001) apresentaram 2,0 e 2,2 maior chance de desenvolver alteração do estado da pele do que RN com IG entre 37 e 41 semanas e $\mathrm{RN}$ com peso $>2.500$ gramas, respectivamente. Quanto ao crescimento intrauterino, RN classificado como Pequeno para Idade Gestacional (IC 95\% 1,0 - 79,5; $\mathrm{p}=0,024)$ demonstrou nove vezes maior chance de apresentar alteração da condição cutânea do que RN enquadrado como Adequado para Idade Gestacional (Tabela 2). No que se refere à idade materna, mães com idades entre 14 e 19 anos (IC 95\% 1,0 - 48,6; p = 0,032 ) e entre 20 e 29 anos (IC 95\% 1,8 - 32,6; p = 0,004) apresentaram, respectivamente, 7,2 e 7,6 maior probabilidade de possuírem recém-nascidos com estado da pele alterado (Tabela 2). 
Tabela 2. Distribuição do número de recém-nascidos, segundo a condição de pele e as características maternas e do RN

\begin{tabular}{|c|c|c|c|c|c|}
\hline Variáveis & $\begin{array}{c}\text { Pele ideal } \\
(n=19) \\
n(\%)\end{array}$ & $\begin{array}{c}\text { Pele } \\
\text { alterada } \\
(\mathrm{n}=27) \\
\mathrm{n}(\%)\end{array}$ & $\mathrm{RC}$ & IC 95\% & p-value* \\
\hline \multicolumn{6}{|l|}{$\begin{array}{l}\text { Idade materna } \\
\text { (ano) }\end{array}$} \\
\hline $14-19$ & $2(25,0)$ & $6(75,0)$ & 7,2 & $1,1-48,6$ & 0,032 \\
\hline $20-29$ & $5(23,8)$ & $16(76,2)$ & 7,6 & $1,8-32,6$ & 0,004 \\
\hline $30-40$ & $12(70,6)$ & $5(29,4)$ & 1 & & \\
\hline Procedência & & & & & 0,926 \\
\hline Interior & $11(40,7)$ & $16(59,3)$ & 1,1 & $0,3-3,4$ & \\
\hline $\begin{array}{l}\text { Capital e região } \\
\text { metropolitana }\end{array}$ & $8(42,1)$ & $11(57,9)$ & 1 & & \\
\hline \multicolumn{6}{|l|}{$\begin{array}{l}\text { Idade gestacional } \\
\text { (semana) }\end{array}$} \\
\hline > 41 (Pós-termo) & $2(100,0)$ & $0(0,0)$ & 2,0 & $1,4-2,7$ & 0,103 \\
\hline < 37 (Pré-termo) & $0(0,0)$ & $10(100,0)$ & 2,0 & $1,4-2,7$ & 0,004 \\
\hline $37-41$ (A termo) & $17(50,0)$ & $17(50,0)$ & 1 & & \\
\hline \multicolumn{6}{|l|}{$\begin{array}{l}\text { Peso ao nascer } \\
\text { (grama) }\end{array}$} \\
\hline $1.785-2.499$ & $0(0,0)$ & $11(100,0)$ & 2,2 & $1,5-3,1$ & 0,001 \\
\hline $2.500-4.690$ & $19(54,3)$ & $16(45,7)$ & 1 & & \\
\hline \multicolumn{6}{|l|}{$\begin{array}{l}\text { Crescimento } \\
\text { intrauterino }\end{array}$} \\
\hline $\mathrm{PIG} \neq$ & $1(10,0)$ & $9(90,0)$ & 9,0 & $1,1-79,5$ & 0,024 \\
\hline GIG + & $2(50,0)$ & $2(50,0)$ & 1,0 & $0,1-7,9$ & 1,000 \\
\hline AIG $\S$ & $16(50,0)$ & $16(50,0)$ & 1 & & \\
\hline Apgar (10 min.) & & & & & 0,712 \\
\hline $\begin{array}{l}\text { Asfixia grave ou } \\
\text { moderada }\end{array}$ & $2(50,0)$ & $2(50,0)$ & 0,6 & $0,0-5,3$ & \\
\hline Boa vitalidade & $17(40,5)$ & $25(59,5)$ & 1 & & \\
\hline Apgar (5º min.) & & & & & 0,772 \\
\hline $\begin{array}{l}\text { Asfixia grave ou } \\
\text { moderada }\end{array}$ & $1(33,3)$ & $2(66,7)$ & 1,4 & $0,1-17,1$ & \\
\hline Boa vitalidade & $18(41,9)$ & $25(58,1)$ & 1 & & \\
\hline $\operatorname{Sexo}(n=45) / /$ & & & & & 0,872 \\
\hline Feminino & $9(40,9)$ & $13(59,1)$ & 1,1 & $0,3-3,6$ & \\
\hline Masculino & $10(43,5)$ & $13(56,5)$ & 1 & & \\
\hline Idade do RN (dia) & & & & & 0,074 \\
\hline$\leq 7$ & $2(18,2)$ & $9(81,8)$ & 4,2 & $0,8-22,5$ & \\
\hline 8 a 28 & $17(48,6)$ & $18(51,4)$ & 1 & & \\
\hline $\begin{array}{l}\text { Tempo de } \\
\text { internação (dia) }\end{array}$ & & & & & 0,729 \\
\hline$\leq 7$ & $11(39,3)$ & $17(60,7)$ & 1,2 & $0,3-4,1$ & \\
\hline 8 a 23 & $8(44,4)$ & $10(55,6)$ & 1 & & \\
\hline Local de origem & & & & & 0,085 \\
\hline Outro q & $13(35,1)$ & $24(64,9)$ & 3,6 & $0,7-17,2$ & \\
\hline Domicílio & $6(66,7)$ & $3(33,3)$ & 1 & & \\
\hline
\end{tabular}

*Razão de verossimilhança + Grande para Idade Gestacional; \# Pequeno para Idade Gestacional; § Adequado paraldade Gestacional; // Excluído RN com genitália ambígua; ๆ Outros locais: Hospital, Maternidade e UTI Neonatal

Nos aspectos clínicos, demonstrou-se que os distúrbios geniturinários apresentaram associação com o estado da pele $(p=0,047)$, o que retratou que os recém-nascidos com esse diagnóstico apresentavam alteração da condição cutânea. Também, observou-se associação entre os diagnósticos clínicos enquadrados na categoria "Outros" e o estado da pele do RN que mostrou melhor prognóstico nesse grupo. Os demais aspectos clínicos e dispositivos terapêuticos em uso durante a internação não apresentaram associação significativa (Tabela 3).

Na ocorrência de lesões de pele, constatou-se que 21(45,6\%) recém-nascidos apresentaram lesões cutâneas, destes, $8,7 \%$ desenvolveram mais de uma lesão. A dermatite por fraldas e descamação foram as mais prevalentes, as quais corresponderam, cada uma, a $24,1 \%$ do total de ocorrências. Consequentemente, os locais mais incidentes dos achados foram os membros inferiores ou superiores e a genitália externa/região perianal, que contabilizaram oito $(27,5 \%)$ e sete $(24,1 \%)$ lesões nesses sítios, respectivamente (Tabela 4).

\section{Discussão}

No contexto de hospitalização, a maioria dos recém-nascidos apresentou alteração no estado da pele. $\mathrm{O}$ uso de dispositivos clínicos relacionados à assistência à saúde aumenta consideravelmente o risco de rupturas cutâneas. ${ }^{(5)}$ A idade também é considerada fator predisponente para o desenvolvimento de lesões. Logo, as populações idosa, pelo envelhecimento, e, neonatal, pelo subdesenvolvimento da pele, apresentam maior risco de alterações, como documentado na literatura. ${ }^{(10)}$

Diante dos fatores que interferiram na condição cutânea, constatou-se que IG, peso ao nascer e crescimento intrauterino apresentaram associação significativa, visto que RNPT, com baixo peso ao nascer e pequeno para idade gestacional demonstraram estado da pele alterado, de acordo com a pontuação obtida na ECPRN. Tal fenômeno, também observado em unidades de cuidados intensivos, pode ser explicado pela insuficiência da função de barreira cutânea decorrente da prematuridade, haja vista haver exponencial relação inversa entre a IG e a perda transepidérmica de água que mede quantitativamente essa função da pele. Deste modo, cuidados específicos com RNPT, como maior espaçamento na frequência dos banhos, uso de emolientes e escolha do antisséptico adequado para realização de procedimentos invasivos, são algumas medidas úteis para potencialização da barreira cutânea. ${ }^{(11,12)}$ 
Tabela 3. Distribuição do número de recém-nascidos, segundo a condição de pele e os aspectos clínicos e de dispositivos terapêuticos em uso durante a internação

\begin{tabular}{|c|c|c|c|c|}
\hline Variáveis & Pele ideal $n(\%)$ & Pele alterada $n(\%)$ & Total n(\%) & p-value* \\
\hline \multicolumn{5}{|l|}{ Diagnóstico clínico } \\
\hline Doenças infecciosas & $9(45,0)$ & $11(55,0)$ & $20(100,0)$ & 0,655 \\
\hline Doenças respiratórias & $6(37,5)$ & $10(62,5)$ & $16(100,0)$ & 0,702 \\
\hline Distúrbios gastrointestinais & $4(40,0)$ & $6(60,0)$ & $10(100,0)$ & 0,925 \\
\hline Icterícia & $2(22,2)$ & $7(77,8)$ & $9(100,0)$ & 0,195 \\
\hline Malformação congênita & $2(28,6)$ & $5(71,4)$ & $7(100,0)$ & 0,457 \\
\hline Síndrome genética & $4(57,1)$ & $3(42,9)$ & $7(100,0)$ & 0,355 \\
\hline Distúrbios geniturinários & $0(0,0)$ & $5(100,0)$ & $5(100,0)$ & 0,047 \\
\hline Distúrbios neurológicos & $1(20,0)$ & $4(80,0)$ & $5(100,0)$ & 0,305 \\
\hline Trauma & $0(0,0)$ & $2(100,0)$ & $2(100,0)$ & $0,225+$ \\
\hline Outros & $8(72,7)$ & $3(27,3)$ & $11(100,0)$ & 0,015 \\
\hline \multicolumn{5}{|l|}{ Classes medicamentosas } \\
\hline Suplementos vitamínicos & $10(41,7)$ & $14(58,3)$ & $24(100,0)$ & 0,958 \\
\hline Analgésico/Antiemético & $5(26,3)$ & $14(73,7)$ & $19(100,0)$ & 0,083 \\
\hline Antibiótico & $4(26,7)$ & $11(73,3)$ & $15(100,0)$ & 0,161 \\
\hline Hidratação venosa & $5(62,5)$ & $3(37,5)$ & $8(100,0)$ & 0,180 \\
\hline Anticonvulsivante & $1(25,0)$ & $3(75,0)$ & $4(100,0)$ & $0,488+$ \\
\hline Antirretroviral & $1(50,0)$ & $1(50,0)$ & $2(100,0)$ & 0,798 \\
\hline Protetor gástrico & $1(50,0)$ & $1(50,0)$ & $2(100,0)$ & $0,798+$ \\
\hline Antifúngico & $0(0,0)$ & $4(100,0)$ & $4(100,0)$ & $0,079+$ \\
\hline Outras & $3(33,3)$ & $6(66,7)$ & $9(100,0)$ & 0,588 \\
\hline Tratamento não medicamento & $18(42,9)$ & $24(57,1)$ & $42(100,0)$ & 0,488 \\
\hline \multicolumn{5}{|l|}{ Via de medicação } \\
\hline Via Oral (V0) & $7(33,3)$ & $14(66,7)$ & $21(100,0)$ & 0,314 \\
\hline Endovenosa (EV) & $9(45,0)$ & $11(55,0)$ & $20(100,0)$ & 0,655 \\
\hline $\begin{array}{l}\text { Sonda Orogástrica / Sonda } \\
\text { Oroentérica }\end{array}$ & $4(50,0)$ & $4(50,0)$ & $8(100,0)$ & 0,583 \\
\hline Tópica & $1(14,3)$ & $6(85,7)$ & $7(100,0)$ & 0,115 \\
\hline Intramuscular (IM) & $1(25,0)$ & $3(75,0)$ & $4(100,0)$ & 0,488 \\
\hline \multicolumn{5}{|l|}{ Suporte nutricional } \\
\hline vo & $17(47,2)$ & $19(52,8)$ & $36(100,0)$ & 0,122 \\
\hline SOG/SOE/SNE & $6(46,2)$ & $7(53,8)$ & $13(100,0)$ & 0,675 \\
\hline Nutrição Parenteral (NPT) & $0(0,0)$ & $2(100,0)$ & $2(100,0)$ & $0,225+$ \\
\hline Zero & $0(0,0)$ & $3(100,0)$ & $3(100,0)$ & $0,133+$ \\
\hline Fototerapia & & & & $0,504 \neq$ \\
\hline Bilitron simples & $0(0,0)$ & $2(100,0)$ & $2(100,0)$ & \\
\hline Sem fototerapia & $19(43,2)$ & $25(56,8)$ & $44(100,0)$ & \\
\hline Oxigenação & & & & $0,999 \neq$ \\
\hline Ar ambiente & $19(43,1)$ & $25(56,8)$ & $44(100,0)$ & \\
\hline Cânula nasofaríngea & $0(0,0)$ & $1(100,0)$ & $1(100,0)$ & \\
\hline Cateter nasal & $0(0,0)$ & $1(100,0)$ & $1(100,0)$ & \\
\hline Acesso intravenoso & & & & 0,584 \\
\hline Sem acesso & $9(37,5)$ & $15(62,5)$ & $24(100,0)$ & \\
\hline Com acesso $\S$ & $10(45,5)$ & $12(54,5)$ & $22(100,0)$ & \\
\hline Drenos e ostomias & & & & $0,999 \neq$ \\
\hline Sem drenos e ostomias & $18(41,8)$ & $25(58,1)$ & $43(100,0)$ & \\
\hline Colostomia // & $1(33,3)$ & $2(66,6)$ & $3(100,0)$ & \\
\hline
\end{tabular}

*Qui-Quadrado; † Razão de verossimilhança; キ Fisher-Freeman-Halton; § Tipos de acessos intravenosos: Acesso Venoso Periférico; Acesso Venoso Central; Cateter Central de Inserção Periférica; // Durante a coleta de dados, avaliou-se a presença dos diversos tipos de drenos e ostomias, porém observou-se apenas a presença de colostomia em 3 recém-nascidos 
Tabela 4. Ocorrência de lesões na pele dos recém-nascidos e fatores relacionados

\begin{tabular}{lc}
\hline Variáveis & $\mathrm{n}(\%)$ \\
\hline Quantidade de lesões por RN $(\mathrm{n}=46)$ & $25(54,3)$ \\
Nenhuma & $17(37,0)$ \\
1 & $4(8,7)$ \\
2 ou mais & \\
Tipo ( $\mathrm{n}=29)$ & $7(24,1)$ \\
Dermatite por fraldas & $7(24,1)$ \\
Descamação & $5(17,2)$ \\
Incisão cirúrgica & $3(10,3)$ \\
Eritema localizado & $2(6,8)$ \\
Vesícula / Pústula & $2(6,8)$ \\
Lesão por extravasamento & $1(3,4)$ \\
Hematoma & $1(3,4)$ \\
Flebite & $1(3,4)$ \\
Onfalocele & \\
Local ( $\mathrm{n}=29)$ & $2(6,9)$ \\
Face & $8(27,6)$ \\
Membros inferiores ou superiores & $2(6,9)$ \\
Tórax anterior & $7(24,1)$ \\
Genitália externa e região perianal & $6(20,7)$ \\
Abdômen & $3(10,3)$ \\
Região umbilical & $1(3,5)$ \\
Localização difusa &
\end{tabular}

A idade materna inferior a 30 anos também foi associada ao pior estado da pele do RN. A prematuridade pode estar associada a essa condição, haja vista maior incidência de partos prematuros em genitoras com faixa etária inferior a 20 anos, bem como naquelas com pré-natal insatisfatório e ocorrência de parto cesáreo. $^{(13)}$

Ao analisar os fatores maternos relacionados às manifestações cutâneas no RN, estudo italiano também concluiu que recém-nascidos hospitalizados de mães primigestas têm maior frequência de lesões na pele fisiológicas do que de mães multigestas. ${ }^{(14)}$

No que tange aos diagnósticos clínicos, recém-nascidos com distúrbios geniturinários apresentaram estado da pele alterado. A população neonatal apresenta, em decorrência do menor peso corporal, maior tendência à desidratação. Somado a esse fator, o déficit no estado hídrico é um achado clínico comum em crianças hospitalizadas com disfunções renais. Tais condições favorecem o prejuízo da barreira cutânea, uma vez que a hidratação desempenha função vital na manutenção da integridade da pele. ${ }^{(10,15,16)}$

Em contrapartida, apesar das evidências científicas, ainda que insuficientes, apontarem que o uso de determinadas classes farmacológicas como corticoste- roides, pelo efeito inibidor da síntese de colágeno, e anticoagulantes, considerado agente etiológico para equimoses, ser fator que interfere na integridade cutânea, nenhuma classe medicamentosa utilizada pelos recém- nascidos demonstrou associação com a condição da pele apresentada. ${ }^{(10)}$

O período de internação não apresentou associação com o estado cutâneo. Em estudo que envolveu recém-nascidos internados em unidade de terapia intensiva neonatal, o tempo de permanência hospitalar inferior a sete dias fora mais prevalente igualmente à UCINCo avaliada. No ambiente de cuidados intensivos, porém, evidenciou-se que os recém-nascidos com período superior a sete dias de internação obtiveram pontuação maior na ECPRN quanto ao quesito secura, tal fato não fora observado na unidade estudada. ${ }^{(11)}$

Achados cutâneos, geralmente benignos e autolimitados, são incidentes na população neonatal. ${ }^{(17)}$ Assim como um estudo que analisou a ocorrência de lesões de pele em uma unidade de neonatologia, a lesão mais prevalente, juntamente à descamação superficial da pele, foi a dermatite por fraldas que atinge amplamente os recém-nascidos. Especialmente na região das fraldas, a pele neonatal apresenta maior vulnerabilidade a rupturas. O contato direto com eliminações vesico intestinais, fricção, colonização microbiana e produtos químicos propendem ao surgimento da dermatite. Deste modo, a troca frequente de fraldas, associada ao uso de creme de barreira adequado, e, quando possível, de fraldas superabsorventes, são cuidados importantes para prevenção e tratamento desses eventos. ${ }^{(18,19)}$

Em contrapartida, no âmbito de cuidados intensivos neonatais, observa-se maior incidência de escoriações decorrentes da fixação de dispositivos clínicos, lesão por pressão associada a tais artigos terapêuticos e queimaduras. Diferentemente das unidades de cuidados intermediários, tais achados decorrem da necessidade prolongada de uso de sondas orogástricas e cânulas orotraqueais, além da monitorização contínua por oximetria de pulso do $\mathrm{RN}$ em unidades intensivas. ${ }^{(6)}$

Além disso, apesar de não haver associação significativa entre a condição da pele e a presença de acesso venoso, identificou-se que alguns recém-nascidos com este dispositivo possuíam alterações cutâneas decorrentes de flebite e lesão por extrava- 
samento, achado importante na população neonatal hospitalizada. Tal evento apresenta como fatores predisponentes a IG, o peso ao nascer, a antibioticoterapia e a nutrição parenteral. Deste modo, ao constatar esses fatores, os profissionais devem estar aptos a documentar e avaliar regularmente o sítio de inserção do cateter, além de identificar e implementar, precocemente, medidas cabíveis frente a essa complicação. ${ }^{(20)}$

Por fim, identificou-se, como limitação do presente estudo, a ausência de reavaliações da pele do RN, haja vista ser análise de corte transversal, a qual impossibilita quantificar o surgimento de lesões cutâneas durante a internação. Além dessa insuficiência, durante o período de coleta de dados, parcela das crianças internadas na UCINCo ultrapassava a faixa etária correspondente ao período neonatal, já que a unidade assiste lactentes até três meses, fato que gerou redução da amostra analisada.

Porém, os resultados obtidos são de grande relevância, visto que retratam os fatores relacionados à condição da pele do RN em unidade de cuidados intermediários e completam lacunas na literatura, uma vez que são escassos estudos acerca do tema nessas unidades.

\section{Conclusão}

Identificaram-se frequentes as alterações cutâneas na população analisada, uma vez que $58,7 \%$ dos recém-nascidos apresentaram estado da pele alterado. Este fenômeno apresenta fatores predisponentes importantes, como presença de distúrbios geniturinários no $\mathrm{RN}$, idade materna $<30$ anos, baixo peso ao nascer, pequeno para idade gestacional e IG $<37$ semanas (prematuridade), estatisticamente comprovados. Desse modo, diante da identificação desses fatores na prática assistencial, sugere-se a implementação de cuidados específicos à pele do $\mathrm{RN}$, de modo a garantir o uso de produtos antissépticos adequados e adaptação do horário para banhos, além de alertar enfermeiros sobre a importância da constante avaliação da condição da pele, para prevenir complicações associadas às alterações cutâneas durante a internação.

\section{Contribuições}

Sales JMR, Lima FET, Almeida PC, Oliveira RMC e Santos IB contribuíram com a concepção do estudo, análise e interpretação dos dados, redação do artigo, revisão crítica relevante do conteúdo intelectual e aprovação da versão final a ser publicada.

\section{Referências}

1. Liu L, Oza S, Hogan D, Chu Y, Perin J, Zhu J, et al. Global, regional, and national causes of under-5 mortality in 2000-15: an updated systematic analysis with implications for the Sustainable Development Goals. Lancet. 2016;388(10063):3027-35.

2. Brasil. Ministério da Saúde. Departamento de Informática do SUS (DATASUS). Informações de Saúde (TABNET) - Estatísticas Vitais - Óbitos Infantis. Brasília (DF): Ministério da Saúde; 2017. [citado 2021 Ago 10]. Disponivel em: http://tabnet.datasus.gov.br/cgi/deftohtm. exe? sim/cnv/inf10uf.def

3. Dyer JA. Newborn skin care. Semin Perinatol. 2013;37(1):3-7.

4. Sodré CT, Azulay DR, Azulay RD. A Pele - Função, Estrutura, Fisiologia e Embriologia. 7a ed. Rio de Janeiro: Guanabara Koogan; 2017.

5. Schaefer TI, Naidom AM, Neves ET. Skin care to newborns admitted in neonatal intensive care unit: integrative review. Rev Fund Care. 2016;8(4):5156-62.

6. Faria MF, Ferreira MB, Felix MM, Calegari IB, Barbosa MH. Factors associated with skin and mucosal lesions caused by medical devices in newborns: Observational study. J Clin Nurs. 2019;28(21-22):3807-16

7. García-Molina P, Balaguer-López E, García-Fernández FP, Ferrera-Fernández ML, Blasco $J M$, Verdú J. Pressure ulcers' incidence, preventive measures, and risk factors in neonatal intensive care and intermediate care units. Int Wound J. 2018;15(4):571-9.

8. Aredes ND, Santos RC, Fonseca LM. Cuidados com a pele do recém-nascido prematuro: revisão integrativa. Rev Eletrônica Enferm. 2017;19:1-25.

9. Schardosim JM. Adaptação transcultural e validação clínica do instrumento neonatal Skin Condition Score para uso no Brasil [dissertação]. Porto Alegre: Escola de Enfermagem, Universidade Federal do Rio Grande do Sul; 2012.

10. Serra R, lelapi N, Barbetta A, Franciscis S. Skin tears and risk factors assessment: a systematic review on evidence-based medicine. Int Wound J. 2018;15(1):38-42.

11. Schaefer TI, Neves ET, Jantsch LB, Magnago TS. Avaliação das condições da pele do recémnascido em terapia intensiva neonatal. Rev Enferm Atual. 2018;84:33-44.

12. Kusari A, Han AM, Virgen CA, Matiz C, Rasmussen M, Friedlander SF, et al. Evidence- based skin care in preterm infants. Pediatr Dermatol. 2019;36(1):16-23.

13. Oliveira LL, Gonçalves AC, Costa JS, Bonilha AL. Maternal and neonatal factors related to prematurity. Rev Esc Enferm USP. 2016;50(3):382-9.

14. Giuffrida R, Borgia F, Pasquale L, Guarneri F, Cacace C, Cannavò SP. Skin lesions in preterm and term newborns from Southern Italy and their relationship to neonatal, parental and pregnancy-related variables. G Ital Dermatol Venereol. 2019;154(4):400-4.

15. Chami N, Kabyemera R, Masoza T, Ambrose E, Kimaro F, Kayange $\mathrm{N}$, et al. Prevalence and factors associated with renal dysfunction in children admitted to two hospitals in northwestern Tanzania. BMC Nephrol. 2019; 20(1):79.

16. Saghaleini SH, Dehghan K, Shadvar K, Sanaie S, Mahmoodpoor A, Ostadi Z. Pressure ulcer and nutrition. Indian J Crit Care Med. 2018;22(4):283-9.

17. Rayala BZ, Morrell DS. Common Skin Conditions in Children: Neonatal Skin Lesions. FP Essent. 2017;453:11-7.

18. Migoto MT, Souza SN, Rossetto EG. Skin lesions of newborns in a neonatal unit: descriptive study. Online Braz J Nurs. 2013;12(2):377-92.

19. Burdall 0 , Willgress L, Goad N. Neonatal skin care: Developments in care to maintain neonatal barrier function and prevention of diaper dermatitis. Pediatr Dermatol. 2019;36(1):31- 5 .

20. Atay S, Sen S, Cukurlu D. Incidence of infiltration/extravasation in newborns using peripheral venous catheter and affecting factors. Rev Esc Enferm USP. 2018;52:e03360. 